\title{
Upper Ocean Turbulence from High-Resolution 3D Simulations
}

\author{
Patrice Klein and Bach Lien Hua \\ Laboratoire de Physique des Océans, IFREMER/CNRS, Brest, France
}

GuILLAUME LAPEYRE

Laboratoire de Météorologie Dynamique/IPSL, Ecole Normale Supérieure/CNRS, Paris, France

XAvier CAPET

Institute of Geophysics and Planetary Physics, University of California, Los Angleles, Los Angeles, California

SYLVIE LE GENTIL

Laboratoire de Physique des Océans, IFREMER/CNRS, Brest, France

HIDEHARU SASAKI

Earth Simulator Center, JAMSTEC, Yokohama, Japan

(Manuscript received 29 January 2007, in final form 17 December 2007)

\begin{abstract}
The authors examine the turbulent properties of a baroclinically unstable oceanic flow using primitive equation (PE) simulations with high resolution (in both horizontal and vertical directions). Resulting dynamics in the surface layers involve large Rossby numbers and significant vortical asymmetries. Furthermore, the ageostrophic divergent motions associated with small-scale surface frontogenesis are shown to significantly alter the nonlinear transfers of kinetic energy and consequently the time evolution of the surface dynamics. Such impact of the ageostrophic motions explains the emergence of the significant cyclone-anticyclone asymmetry and of a strong restratification in the upper layers, which are not allowed by the quasigeostrophic (QG) or surface quasigeostrophic (SQG) theory. However, despite this strong ageostrophic character, some of the main surface properties are surprisingly still close to the surface quasigeostrophic equilibrium. They include a noticeable shallow $\left(\approx k^{-2}\right)$ velocity spectrum as well as a conspicuous local spectral relationship between surface kinetic energy, sea surface height, and density variance over a large range of scales (from 400 to $4 \mathrm{~km}$ ). Furthermore, surface velocities can be remarkably diagnosed from only the surface density using SQG relations. This suggests that the validity of some specific SQG relations extends to dynamical regimes with large Rossby numbers. The interior dynamics, on the other hand, strongly differ from the surface dynamics, involving a small Rossby number, a steep $\left(\approx k^{-4}\right)$ velocity spectrum, and a somewhat steeper density spectrum. The compensation of the surface restratification by a destratification at depth confirms a connection between the surface and the interior induced by the small-scale divergent motions.
\end{abstract}

\section{Introduction}

Mesoscale (100-300 km) oceanic eddies (the ocean's weather systems) are ubiquitous features in infrared and color satellite images (Abraham et al. 2000). A

Corresponding author address: P. Klein, LPO, IFREMER, BP 70, 29280 Plouzané, France.

E-mail: pklein@ifremer.fr renewed interest has been stimulated by results from Hurlburt and Hogan (2000) and Siegel et al. (2001), which significantly strengthen the vision of an upper ocean crowded with a large number of strongly interacting eddies. Their numerical experiments in the North Atlantic basin have shown that a horizontal resolution of $1.5 \mathrm{~km}$ leads to an explosion of eddies, with an eddy kinetic energy (KE) increase by a factor of 10 compared with "eddy-permitting-resolving" (with a 15-

DOI: $10.1175 / 2007 J P O 3773.1$ 
km resolution) simulations (Hurlburt and Hogan 2000; Siegel et al. 2001). However, results of these simulations are close to quasi-2D turbulence because they use only six vertical levels. Thus, one question is as follows: What is the sensitivity to the vertical resolution of the dynamical impact of the submesoscale structures (with scales equal or smaller than $20 \mathrm{~km}$ ) on the ocean dynamics? Motivation for this question is that nonlinear dynamics of mesoscale and submesoscale structures are characterized by a Burger number value close to 1 (a generic property for balanced flows), which means that horizontally thin structures are also vertically thin. Therefore, an accurate representation of their nonlinear dynamics (in particular that related to surface frontogenesis) requires taking into account their aspect ratios.

Mesoscale eddy and submesoscale turbulence has been thoroughly studied within the framework of quasigeostrophic (QG) theory based on a small Rossby number approximation ${ }^{1}$ (Charney 1971; Hua and Haidvogel 1986; Rhines 1979; Smith and Vallis 2001). Increasing the spatial resolution in the three directions does not change significantly the properties highlighted by these turbulence studies (McWilliams 1989). These properties include a steep velocity spectrum (close to $k^{-3}$ or $k^{-4}$ ), a direct cascade of baroclinic (or potential) energy from large to small scales, and an inverse barotropic energy cascade from scales close to the Rossby radius of deformation to larger scales (Held and Larichev 1996; Salmon 1980). Smith and Vallis (2001) further showed that surface-intensified stratification slows the transition from the baroclinic mode to the barotropic mode (through baroclinic instability) and suggested that resulting surface-intensified motions are principally captured by the first baroclinic mode. All of these studies, however, assume zero surface density perturbation.

Considering nonzero surface density perturbations [as in primitive equation (PE) simulations] leads to different dynamics whose representation is much more sensitive to the spatial resolution. Indeed, surface density cascades down to small scales, leading to smallscale surface frontogenesis. The latter mechanism is not restrained because the vertical velocity is zero there. Within this context, Blumen (1978), using the small Rossby number approximation, introduced the surface quasigeostrophic system (SQG), which serves as a counterpart of the model of geostrophic turbulence of Charney (1971). The SQG system involves the same

\footnotetext{
${ }^{1}$ The Rossby number can be defined as the RMS value of the relative vorticity divided by the Coriolis parameter $f$.
}

assumptions as the quasigeostrophic (QG) system, in particular, the small Rossby number approximation. In addition, it considers a nonzero surface density and assumes a uniform potential vorticity (PV) in the interior. The consequence is that its three-dimensional dynamics (and in particular the vertical structure of the surface fronts) are entirely driven by the nonzero surface density using analytical relations (see the appendix). The SQG properties differ from those for geostrophic turbulence. They involve a shallow $\left(k^{-5 / 3}\right)$ surface velocity spectrum (i.e., with more energetic small scales than geostrophic turbulence) identical to the density spectrum (when appropriately scaled). The SQG dynamics have been increasingly proven to be relevant to describe the dynamics of the upper troposphere (Juckes 1994; Tulloch and Smith 2006) and of the upper ocean (Lapeyre and Klein 2006). Thus, Lapeyre and Klein (2006), using the invertibility of potential vorticity, show that the dynamics can be decomposed in terms of a solution forced by the potential vorticity in the interior (QG mode) and a solution forced by the surface density (SQG mode). Relevance of their analysis was successfully demonstrated by PE numerical simulations of a nonlinear baroclinic unstable flow in free decay. They used a coarse resolution $(6 \mathrm{~km}$ in the horizontal direction), which explains their resulting small mean Rossby number (0.06); but their vertical resolution (33 vertical levels) was high enough to represent the SQG mode.

Limitation of SQG theory, however, quickly appears when the horizontal resolution used is high. Indeed, surface density within a filament is conserved in SQG theory, but the vorticity magnitude increases as the filament is stretched and thinned (Held et al. 1995). This means that when the resolution is high enough, smallscale values of the relative vorticity may approach $f$ leading to Rossby numbers on the order of one in these submesoscales. Surface flows with such large Rossby numbers depart from SQG and are principally akin to ageostrophic (or finite time) frontogenesis. ${ }^{2}$ In these situations representation of the flow dynamics requires the use of more complex systems, such as the semigeostrophic (SG) theory or the PE system, which take into account critical nonlinear interactions with large Rossby numbers. These nonlinear interactions involve advection of the geostrophic quantities not only by the geostrophic motions but also by ageostrophic motions,

\footnotetext{
${ }^{2}$ Ageostrophic frontogenesis is characterized by a much faster frontal development than QG frontogenesis (described by the SQG theory) because of the advection of geostrophic momentum by the of $O(1)$ ageostrophic motions (Hoskins 1975). Such ageostrophic frontogenesis is not allowed by SQG.
} 
which is not allowed by the SQG or QG theory. Andrews and Hoskins (1978) and Blumen (1978), using SG theory, showed that the resulting ageostrophic surface frontogenesis leads to a steeper velocity spectrum (with a $k^{-8 / 3}$ slope) than in SQG. In addition, Blumen (1978) indicates that in SG theory the surface density variance is no longer conserved as it is in SQG theory: fluctuations of the divergence at the frontal discontinuities may affect the magnitude of the total surface density variance.

Recently, Hakim et al. (2002) further explored the surface dynamics with large Rossby numbers by using an SQG+1 model, which, in addition to the SQG model, allows the advection of geostrophic quantities by the ageostrophic velocities. This model was developed to understand and explore the vortical asymmetries observed on the tropopause, which SQG theory fails to reproduce. Their results, obtained with a high horizontal resolution (vertical structure in the $\mathrm{SQG}+1$ model-as in the SQG model-is deduced analytically), show that the divergence associated with the small-scale ageostrophic frontogenesis hastens the contraction of denser filaments and slows that of lighter filaments. They argue that these filament asymmetries trigger in their simulations vortical asymmetries with strong localized mesoscale cyclones and weaker diffuse anticyclones as well as a significant global restratification at the tropopause surface. Such surface restratification was also found in the PE simulations of Lapeyre et al. (2006), which use a coarse 3D resolution as in Lapeyre and Klein (2006) but also a much stronger baroclinic forcing [which explains their relatively large mean Rossby number (0.3)]. These authors argue, using Ertel potential vorticity developments, that their restratification is entirely driven by the surface ageostrophic frontogenesis. Thus, flows with large Rossby numbers experience significant departures from SQG, which result from the divergent motions in small-scale surface fronts.

In this study we present the results of a numerical simulation of ocean turbulence in a large domain performed at a high resolution (both horizontally and vertically) with a PE model. The horizontal resolution (2 $\mathrm{km}$ ) is close to that used by Hurlburt and Hogan (2000), but the vertical resolution involves 100 levels instead of 6 levels. Such consistent 3D resolution is required to resolve the small-scale structures and, in particular, to represent the vertical motions in the upper layers. This simulation involves a realistic parameter settings close to that considered in Lapeyre and Klein (2006), but with a much higher spatial resolution. Furthermore, it is forced by a relaxation to a baroclinically unstable basic state, which allows simulation durations on the order of 600 days instead of 50 days in the free-decay simulation of Lapeyre and Klein (2006). The results are analyzed to quantify the impact of the submesoscale structures (and in particular the small-scale surface fronts)driven by the interactions among mesoscale eddieson the ocean surface dynamics and also on the global ocean properties. A more precise question addressed is how much mesoscale and submesoscale turbulence departs from SQG in the upper layers and what new properties emerge when high resolution is used. The next section describes the numerical simulation performed and presents the resulting general characteristics. The following section analyzes the resemblance of the nearsurface dynamics with SQG, whereas section 4 analyzes the departures of these dynamics from SQG and the consequences for the global dynamics. Conclusions are offered in the last section.

\section{Simulations of eddy turbulence with high 3D resolution}

\section{a. Numerical simulations}

Numerical simulations of a nonlinear baroclinic unstable flow in a zonal $\beta$-plane channel centered at $45^{\circ} \mathrm{N}$, based on the primitive equations code Regional Oceanic Modeling System (ROMS; see Shchepetkin and McWilliams 2005; Shchepetkin 2008), have been performed. The parameter settings for the unstable westerly zonal flow resemble those used by Karsten et al. (2002) and Rivière et al. (2004). Salinity is taken as constant, so that temperature anomalies are proportional to density anomalies. The model is a $\sigma$ coordinate model with free-slip boundary conditions at northern and southern walls. The domain size is 1000 $\mathrm{km} \times 2000 \mathrm{~km}$ and its depth is $4000 \mathrm{~m}$. The numerical resolution is $2 \mathrm{~km} \times 2 \mathrm{~km}$ in the horizontal and 100 levels on the vertical (vertical grid spacing ranges from $3 \mathrm{~m}$ near the surface to $200 \mathrm{~m}$ near the bottom). Horizontal mixing uses a Laplacian scheme with a coefficient of $3 \mathrm{~m}^{2} \mathrm{~s}^{-1}$. Vertical diffusion in the interior uses a $K$-profile parameterization (KPP). ${ }^{3}$ The initial vertical profile of the mean Brunt-Väisälä frequency (Fig. 1a) represents a main thermocline located at a depth around $600 \mathrm{~m}$ and corresponds to a first Rossby radius of deformation of approximately $30 \mathrm{~km}$. No surface mixed layer is initially present $(N / f=8$ at the surface

\footnotetext{
${ }^{3}$ The KPP scheme of Large et al. (1994) includes a parameterization of the mixed-layer dynamics. It also includes a parameterization of the vertical mixing in the ocean interior using a critical Richardson number.
} 
(a)

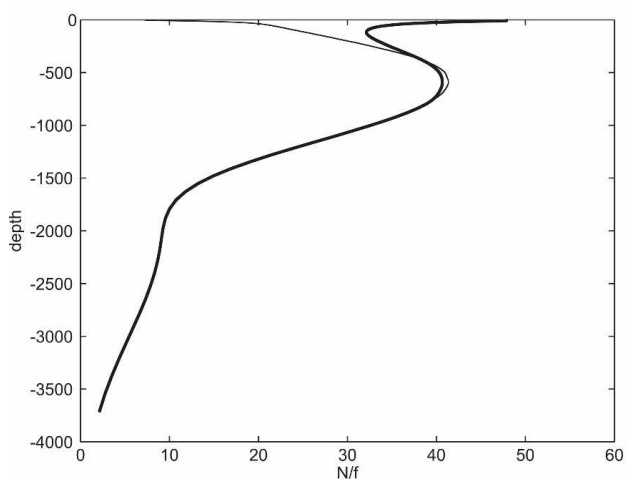

(b)

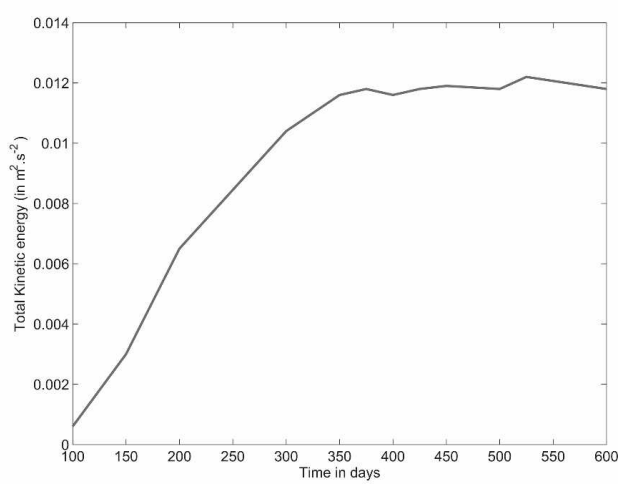

FIG. 1. (a) Vertical profile of $N / f_{0}$ at the initial time (thin curve) and at the equilibrium (thick curve) and (b) time series of the total $\mathrm{KE}\left(\mathrm{m}^{2} \mathrm{~s}^{-2}\right)$.

with $N$ the Brunt-Väisälä frequency and $f$ the Coriolis frequency). This initial state consists of an unstable large-scale meridional density gradient that is surface intensified [in the same manner as Lapeyre and Klein (2006) but weaker in amplitude]. The mesoscale and submesoscale eddy turbulence is forced by using a relaxation of the zonally averaged velocity and density fields to a basic state that corresponds to the initial state. The chosen relaxation time is 50 days. While adjusting the zonal-mean flow, such forcing does not damp the eddies, which helps in maintaining the turbulent eddy field. There is no atmospheric forcing.

After a spinup of 350 days, the total kinetic energy (integrated over the whole domain) has saturated, but its time evolution during the next 300 days is still affected by low-frequency variations with weak amplitudes (Fig. 1b). Results described in the next sections concern the characteristics of the turbulent eddy field near the surface and within the interior well after the spinup period (i.e., between 450 and 600 days).

\section{b. General characteristics}

Figures 2a-c show snapshots of the sea surface height (SSH), density, and relative vorticity fields at the surface. Both SSH and density are characterized by largescale meridional gradients and involve a large number of coherent structures with a size between 20 and 300 $\mathrm{km}$. In addition, the density field exhibits smaller scales and stronger horizontal gradients. The relative vorticity field reveals a much stronger variability at the submesoscales (as small as $8-20 \mathrm{~km}$ ), consisting of numerous small-scale vortices as well as thin filaments, some of which are wavy and hence may be undergoing instability processes. At $800 \mathrm{~m}$, the relative vorticity field (Fig. 2d) displays a spatial heterogeneity-different from that at the surface-and is dominated by larger vortices
$(>100 \mathrm{~km})$ and thicker filaments. This eddy field at 800 $\mathrm{m}$ resembles those from quasigeostrophic turbulence (Hua and Haidvogel 1986; Klein et al. 1998; Larichev and Held 1995; Smith and Vallis 2001).

The difference in properties between the surface layers and the interior is highlighted when examining the vertical profile of dynamical quantities such as kinetic energy and enstrophy at the equilibrium (Figs. 3a,b). A significant surface intensification is observed because kinetic energy is trapped within the first $1000 \mathrm{~m}$, with the maximum located at the surface and almost $50 \%$ of this energy contained within the first $500 \mathrm{~m}$ (Fig. 3a). The much stronger activity at meso- and submesoscales near the surface is emphasized by the vertical profile of the RMS value of the relative vorticity rescaled by $f_{0}$ (the Coriolis frequency at $45^{\circ} \mathrm{N}$ ) (Fig. 3b). This quantity can be interpreted as a mean Rossby number $\left[\sqrt{\left\langle\zeta^{2}\right\rangle} / f_{0}\right.$, with $\zeta$ the relative vorticity equal to $v_{x}-u_{y}$, where $x$ and $y(u$ and $v)$, respectively, denote the zonal and meridional direction (velocity)]. This mean Rossby number is relatively large (close to 0.3 ) near the surface and is much smaller (close to 0.03 ) at $800 \mathrm{~m}$. Relative vorticity extremes reach at the surface $3 f_{0}$ and $-f_{0}$. This lower limit is explained by Haine and Marshall (1998) as resulting from the inertial instability that works for vorticity values smaller than $-f_{0}$. Such vorticity values imply large local Rossby numbers in the upper layers. At $150 \mathrm{~m}$, these extremes are $0.9 f_{0}$ and $-0.45 f_{0}$ and decrease to $\pm 0.4 f_{0}$ at $800 \mathrm{~m}$. Such characteristics indicate the presence of significant ageostrophic processes in the upper layers, whereas the dynamics are close to geostrophy in the interior. One result to note is that the vertical profile of the RMS value of the deformation field (not shown), defined as $\sqrt{\left\langle\sigma_{n}^{2}+\sigma_{s}^{2}\right\rangle} / f_{0}$ (with $\sigma_{n}=$ $u_{x}-v_{y}$ and $\sigma_{s}=v_{x}+u_{y}$ respectively, denoting the normal and shear strain rates), is almost identical to 
(a)

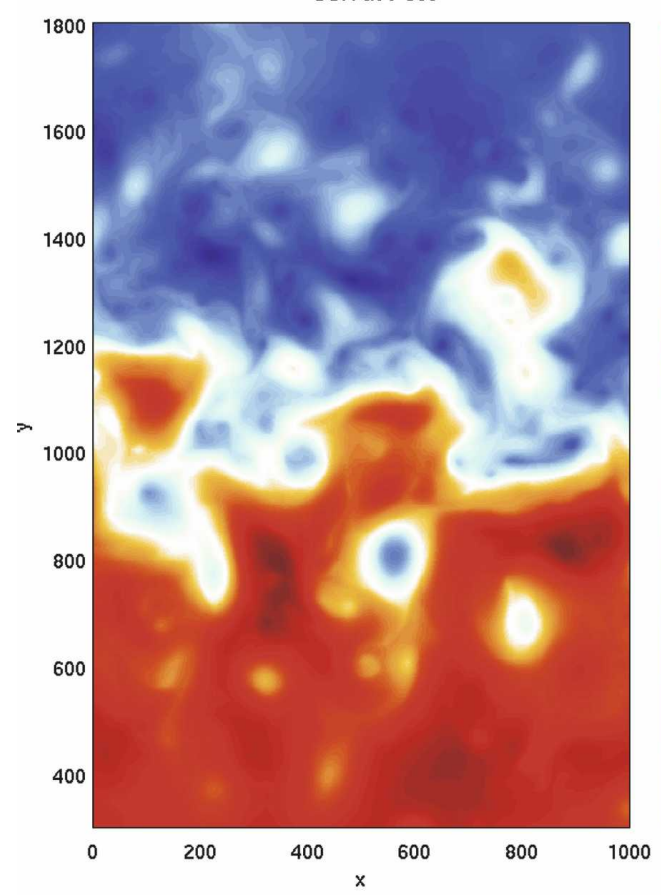

(c)

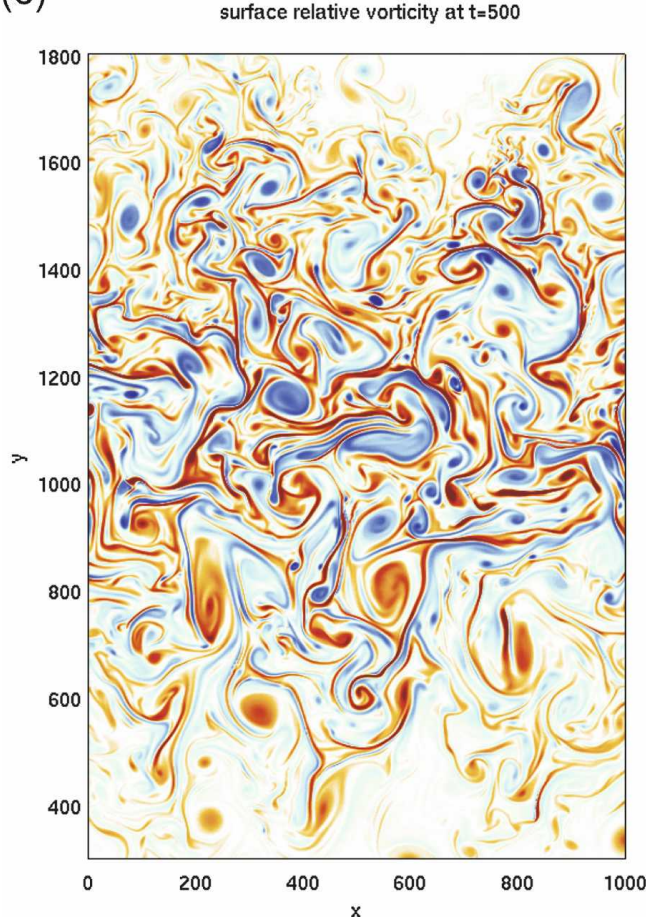

(b)
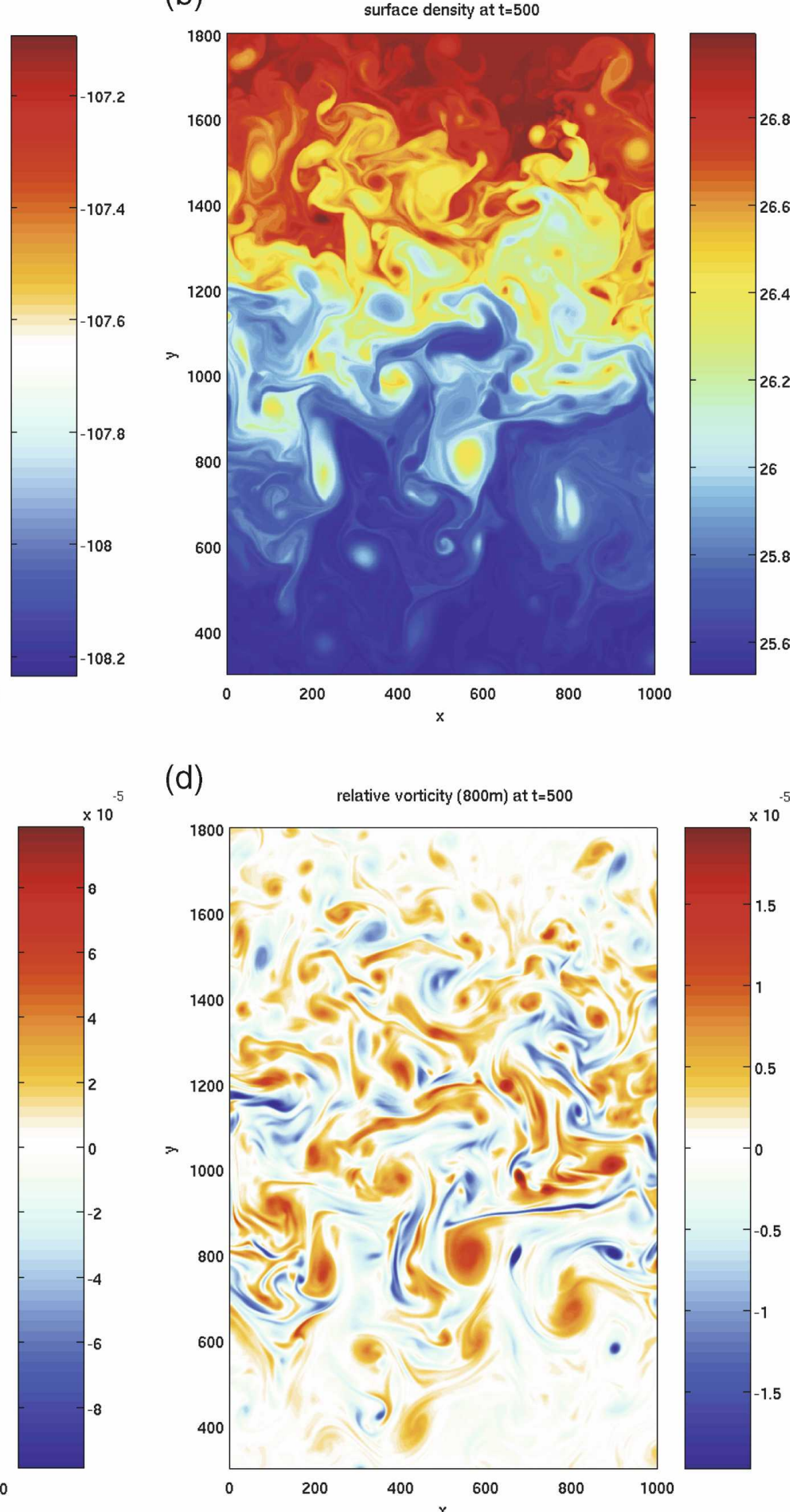

(d)

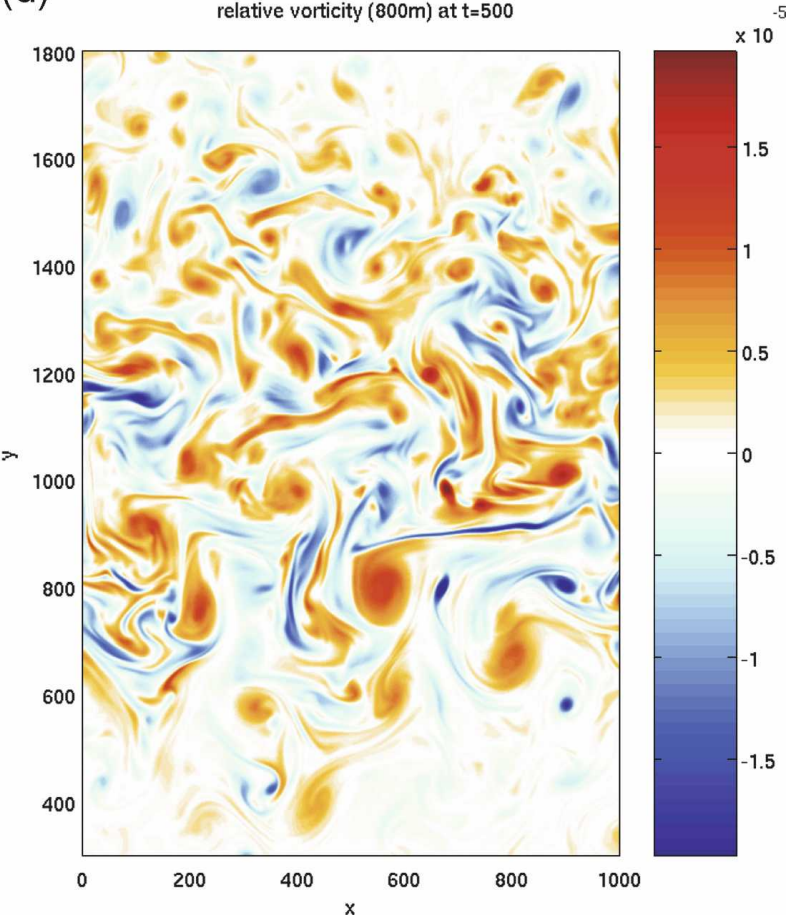

FIG. 2. Snapshots at $t=500$ days of the (a) SSH (m), (b) surface density anomaly $\left(\mathrm{kg} \mathrm{m}^{-3}\right)$, relative vorticity $\left(\mathrm{s}^{-1}\right)$ at the (c) surface and at (d) $800 \mathrm{~m}$. 
(a)

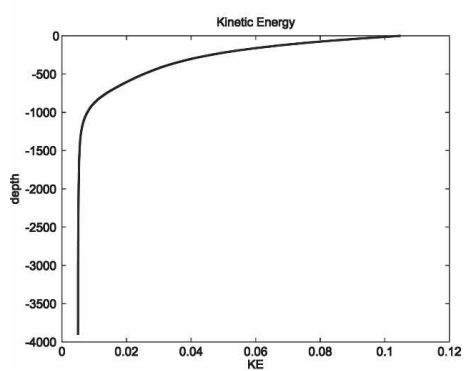

(b)

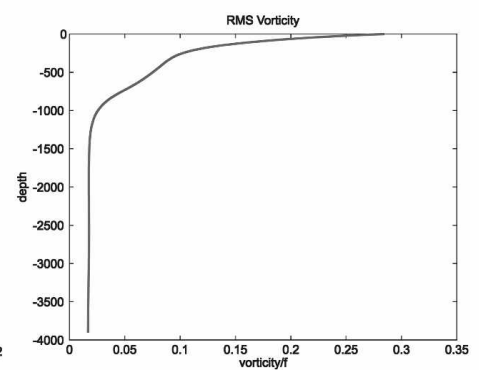

(c)

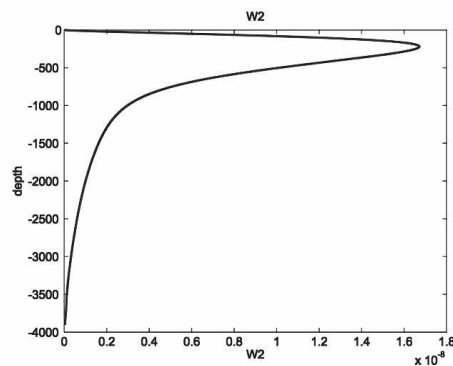

FIG. 3. Vertical profile at $t=500$ days of (a) the kinetic energy $\left(\mathrm{m}^{2} \mathrm{~s}^{-2}\right)$, (b) the RMS value of the relative vorticity normalized by $f_{0}$, and (c) the vertical velocity variance $\left(\mathrm{m}^{2} \mathrm{~s}^{-2}\right)$. Depth is in $\mathrm{m}$.

that for the relative vorticity. This means that the deformation rates are statistically as large as the relative vorticity and display the same contrasts between upper and lower layers. The intermittency in the upper and lower layers is evaluated by the vorticity kurtosis $\left(\left\langle\zeta^{4}\right\rangle /\right.$ $\left\langle\zeta^{2}\right\rangle^{2}$ ). Such a quantity should be close to 3 for Gaussian statistics and has been observed to be 8 to 10 for barotropic and geostrophic turbulence (McWilliams 1984, 1989). From our results, vorticity kurtosis is close to 40 near the surface but is only 6.9 at $600 \mathrm{~m}$.

The probability density function (PDF) of the surface relative vorticity (Fig. 4) exhibits a significant vortical asymmetry: an exponential tail is observed for cyclonic structures and a Gaussian one for anticyclonic structures. The vorticity skewness is close to 2 . The SSH statistics (not shown) display the opposite asymmetry for negative and positive values. Explanations for such vortical asymmetry (Snyder et al. 1991) make use of the gradient wind balance that comes from the divergence of the momentum equations (Hua et al. 1998; McWilliams 1985). This balance is

$$
-\nabla_{H} \cdot\left(\mathbf{u}_{h} \cdot \nabla_{H} \mathbf{u}_{h}\right)+f \zeta=\frac{1}{\rho_{0}} \nabla_{H}^{2} p
$$

where $\boldsymbol{\nabla}_{H}$ is the horizontal gradient operator, $\mathbf{u}_{h}$ is the horizontal velocity vector, $p$ is the pressure, and $\rho_{0}$ is the reference density. The first term in (1) is related to the advective centrifugal force, the second to the Coriolis force, and the last to the pressure gradient force (McWilliams 1985). Within the QG framework, the first term in (1) is equal to $\left(\zeta^{2}-\left\langle\sigma_{n}^{2}+\sigma_{s}^{2}\right\rangle\right) / 2=\mathbf{1} / \rho_{0} \nabla_{H}^{2} p_{a}$, where $p_{a}$ is the ageostrophic pressure and is smaller than the other terms. Hua (1994), within the QG framework, demonstrates that the PDF of this term has a strong asymmetry that resembles that of Fig. 4, whereas the geostrophic pressure PDF is symmetric. Snyder et al. (1991), using a small Rossby number approximation for the primitive equations, show that this term sets up the asymmetry in both the total pressure and the vor- ticity fields. We have evaluated the magnitude of each term in (1) at the surface. In our simulation, the centrifugal term is almost one order of magnitude smaller than the pressure term, except in filaments and some strong small-scale vortices. So the surface flow is on first order remarkably in geostrophic equilibrium, and the vortical asymmetries are principally explained by the asymmetry of the ageostrophic pressure term.

The velocity spectra exhibit significant differences between the upper and lower layers. Near the surface, a noticeable shallow $\left(\approx k^{-2}\right)$ spectrum slope is observed over a large spectral band (see Fig. 5a), implying the presence of energetic small scales there. This spectrum has to be contrasted with the steeper velocity spectrum (with a slope close to $k^{-4}$; Fig. 5d) at $800 \mathrm{~m}$, indicating much less energetic small scales. At this depth, the velocity spectrum compares well with the barotropic ve-

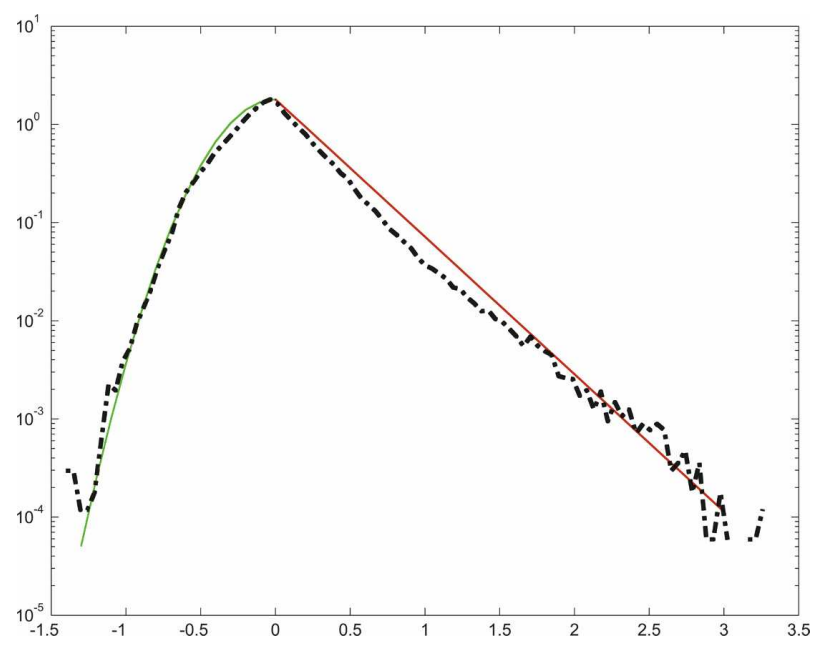

FIG. 4. PDF of surface vorticity (black curve) estimated in the area concerned by the turbulent flow. Exponential law is shown by the red curve and the Gaussian law (with std dev equal to the observed) by the green curve. Abscissa units are vorticity values normalized by $f_{0}$. 
(a)

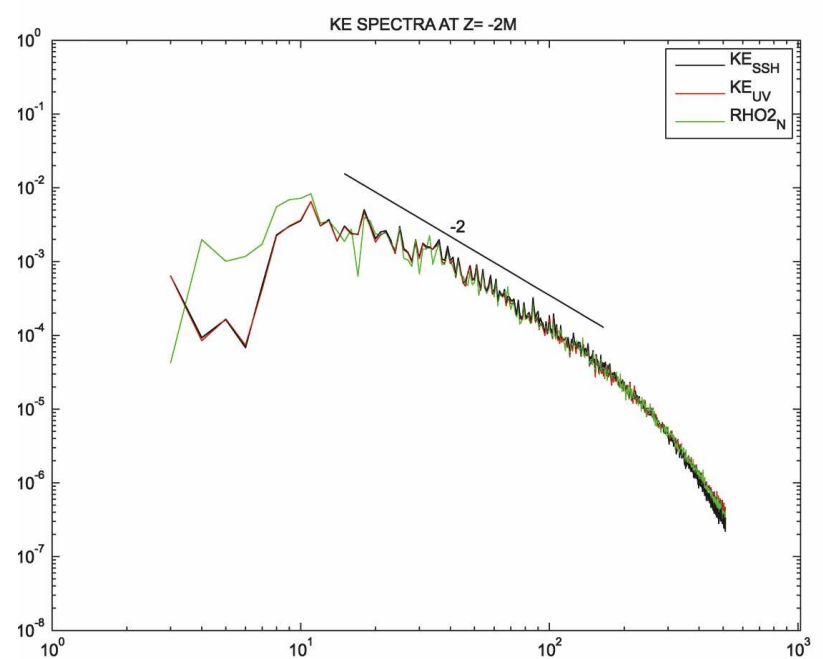

(c)

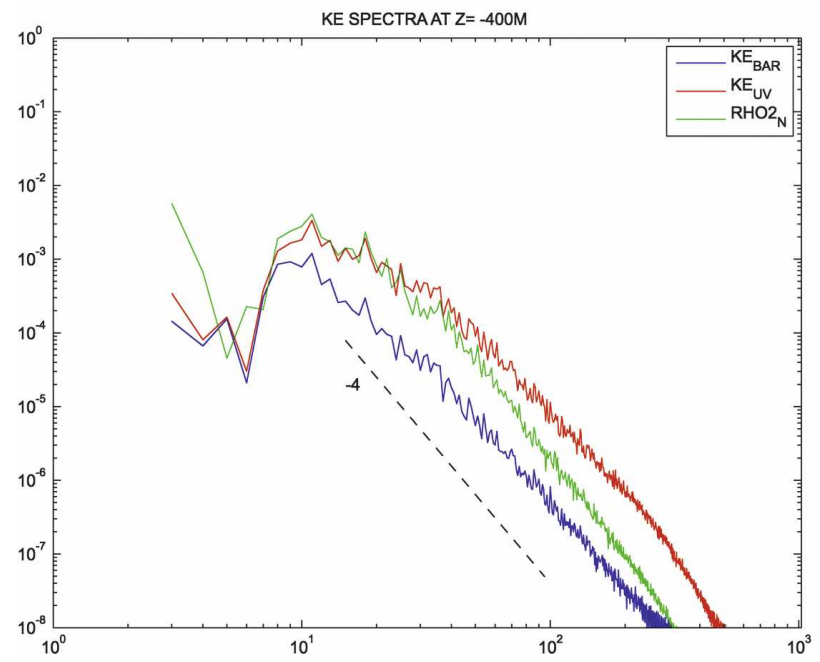

(b)

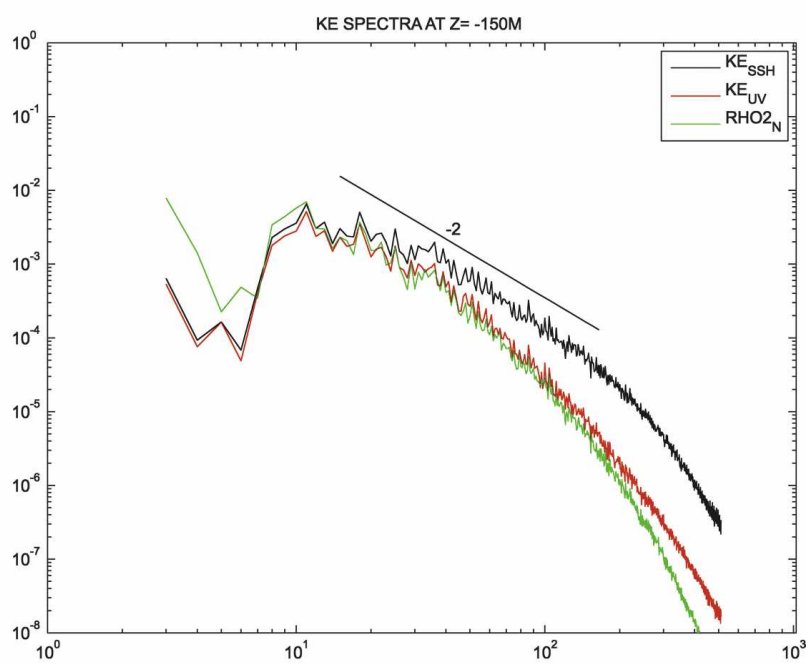

(d)

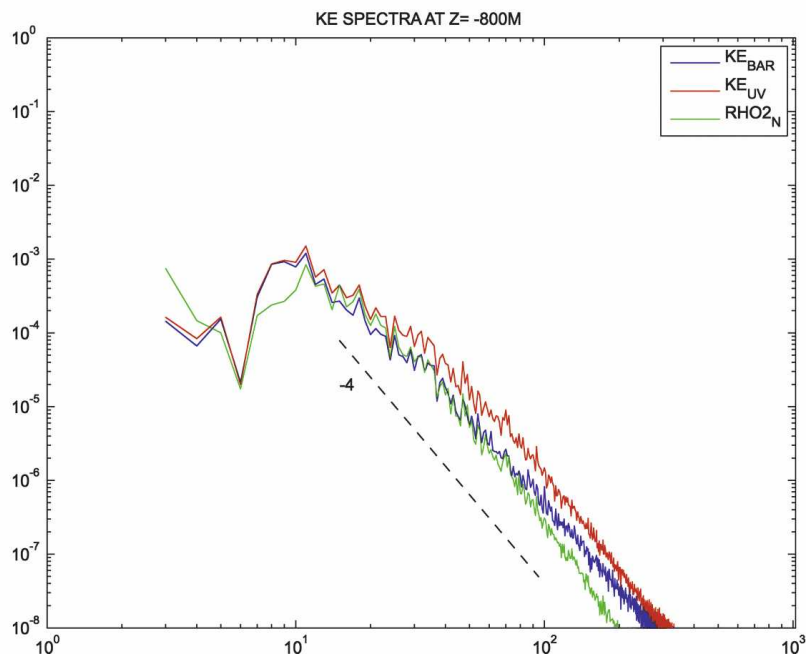

FIG. 5. Velocity spectra estimated from $u$ and $v$ (red curve) at (a) $z=-2 \mathrm{~m}$, (b) $-150 \mathrm{~m}$, (c) $-400 \mathrm{~m}$, and (d) $-800 \mathrm{~m}$. The black curve in (a) and (b) is the surface velocity spectrum estimated from the SSH. The blue curve in (c) and (d) is the velocity spectrum estimated from the barotropic velocity. The green curve in each panel is the density spectrum. Surface density spectrum is scaled by a factor of $g^{2} /\left(N_{0}^{2} \rho_{0}^{2}\right)=7.1$ using $N_{0} / f_{0}=37$ to match the surface velocity spectrum (see text). Other density spectra are scaled to match the velocity spectra at $k=15$. The horizontal axis displays the nondimensional wavenumber $k$. The value $k=10$ corresponds to a wavelength of $300 \mathrm{~km}$. Units on the vertical axis are $\mathrm{m}^{3} \mathrm{~s}^{-2}$.

locity spectrum, suggesting that the energy there is principally captured by this mode.

To quantify the degree of ageostrophy of the flow in the upper layers (where the Rossby number is large) and to further clarify the importance of ageostrophic divergent motions, we have compared the surface velocity spectrum directly calculated from $u$ and $v$ with that estimated from the SSH (using $\mathbf{u}_{h}=-\left(g / f_{0}\right) \mathbf{k} \times$ $\boldsymbol{\nabla} \eta$, where $\eta$ is the SSH and $\mathbf{k}$ is the vertical unit vector). There is an unexpected and remarkable coincidence between both spectra (see Fig. 5a) over the whole spectral range. This confirms the geostrophic equilibrium mentioned before when examining the gradient wind balance, that is, the velocity field near the surface almost equilibrates the pressure field. Then we have estimated the ageostrophic divergent part as the difference between the observed surface horizontal velocity field and its geostrophic part estimated from the SSH. Its spectrum (red curve on Fig. 6) involves a peak around $k=30(100 \mathrm{~km})$. The corresponding spectral amplitudes are, however, much smaller than those for the geostrophic part, except in the small-scale range: in 


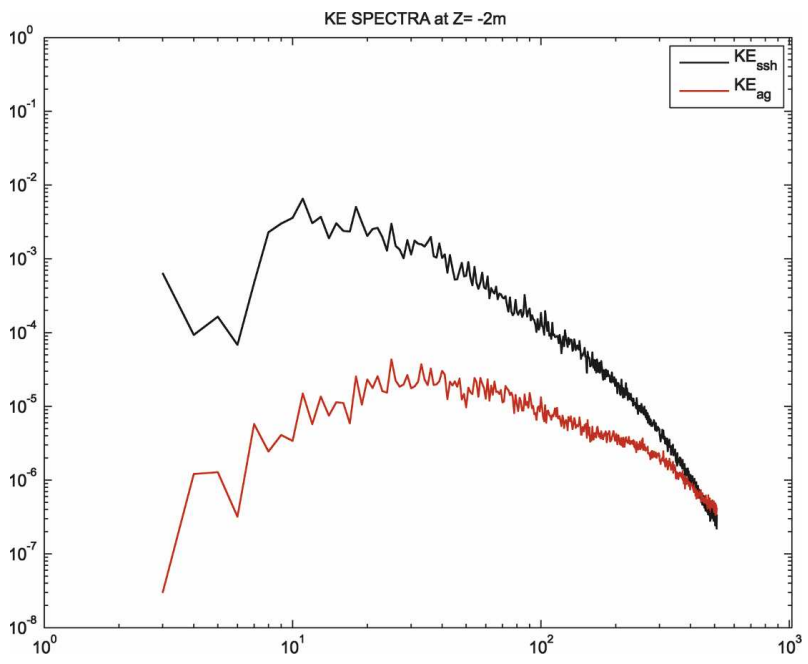

FIG. 6. Spectrum of ageostrophic velocity at the surface (red curve). For comparison, the surface geostrophic velocity spectrum has been plotted (black curve). The horizontal axis displays the nondimensional wavenumber $k$. The value $k=10$ corresponds to a wavelength of $300 \mathrm{~km}$. Units on the vertical axis are $\mathrm{m}^{3} \mathrm{~s}^{-2}$.

terms of velocity, its magnitude compares with that estimated from the SSH only for scales smaller than 20 $\mathrm{km}(k>150)$. Thus, the ageostrophy divergent motions near the surface are energetic principally within the small-scale range, but they do not significantly affect the total horizontal motions (dominated by larger scales).

The picture that emerges from this simulation is the following: on one hand, the lower oceanic layers are characterized by a small Rossby number and therefore the dynamics there appear to be close to the quasigeostrophic equilibrium; on the other hand, characteristics of the upper-layer dynamics are somewhat puzzling. The Rossby number there is large, which emphasizes the significant ageostrophic character at these levels. The result is the presence of a strong vortical asymmetry near the surface, which is absent at depths. Such characteristics are not allowed by QG or SQG theory. However, the surface velocity field has been found to be remarkably in geostrophic equilibrium with the SSH over a large spectral range, and the resulting spectrum has a shallow slope (close to $k^{-2}$ ). This suggests some relationship with the SQG theory. Consequently, we are wondering, in this high-resolution simulation, what part of the upper-layer dynamics verifies the SQG properties and what part clearly departs from SQG and with what important consequences.

\section{Diagnosis of near-surface dynamics from surface density}

The basic property of SQG is that any variable at any depth can be diagnosed from only the surface density.
This comes from the assumption of constant potential vorticity (see appendix). One consequence is that the kinetic energy is identical to the density variance normalized by $g^{2} /\left(N_{0}^{2} \rho_{0}^{2}\right)$. Indeed if $N_{0}$ is assumed to be constant, this can be written as (see the appendix)

$$
\mathrm{KE}_{h}(k, z) \approx \frac{g^{2}}{N_{0}^{2} \rho_{0}^{2}}|\hat{\rho}(k, z)|^{2},
$$

where $\left({ }^{\wedge}\right)$ is the horizontal spectral transform and $\mathrm{KE}_{h}(k, z)=\left[|\hat{u}(k, z)|^{2}+|\hat{v}(k, z)|^{2}\right] / 2$. Here, $g$ is the gravity constant, $N_{0}^{2}$ is the Brunt-Väisälä frequency, and $\rho_{0}=1000 \mathrm{~kg} \mathrm{~m}^{-3}$.

To test whether (2) applies in our simulation, we have plotted on Fig. 5a the density spectrum rescaled by $g^{2} /\left(N_{0}^{2} \rho_{0}^{2}\right)$ using $N_{0} / f_{0}=37$. This value is close to the mean value averaged over $300 \mathrm{~m}(N / f=40)$ observed at the equilibrium (Fig. 1a). Remarkably, the surface velocity and rescaled density spectra are almost identical within a large spectral band (Fig. 5a), between 10 and $400 \mathrm{~km}$ (which corresponds to the spectral peak). This conspicuous similitude in the PE simulation indicates, at least for this range, a local spectral relationship between the amplitudes of these quantities.

It is interesting to see whether (2) still applies at depth. At $800 \mathrm{~m}$ the velocity spectrum slope $\left(k^{-3.5}\right)$ is close to that of the barotropic velocity spectrum, as mentioned before (Fig. 5d). However, the density spectrum (when appropriately scaled at $k=15$ with the velocity spectrum) now well departs from the velocity spectrum for wavenumbers $k>15$ and exhibits a steeper slope $\left(k^{-4.5}\right)$. So SQG cannot be invoked anymore at those depths. These spectral properties in the interior resemble those of QG turbulence reported in Hua and Haidvogel (1986), McWilliams (1989), and Klein et al. (1998). At intermediate depths (Figs. 5b,c), density and velocity spectra reveal a smooth transition between the surface layers and the interior. Indeed, at these depths, these spectra depart from each other for wavenumbers larger than a critical wavenumber, the latter decreasing with depth. In other words, for wavenumbers between the peak wavenumber and this critical wavenumber, the spectral characteristics resemble the surface properties: density and velocity spectra are almost identical. For higher wavenumbers, the density spectrum has a steeper slope than the velocity spectrum.

The smooth transition observed as depth increases is curious but understandable if we refer to the arguments of Lapeyre and Klein (2006) and Scott (2006), corroborated by the previous comment about the aspect ratio of the submesoscale structures. Between the surface and deep layers, the KE amplitude of an SQG mode is known to exponentially decrease with this decrease 
much greater for the smallest horizontal scales. Thus, using the relations of the appendix, the SQG velocity spectrum should display a depth dependence of the form $\overline{\mathrm{KE}_{h}(k, z)}=A k^{-2} \exp \left(2 N_{0} k z / f_{0}\right.$ ) (where $A$ is a constant and $z<0$ ), as also noted by Scott (2006; see also the appendix). On the other hand, the slope of the velocity spectrum associated with the interior mode keeps almost constant as depth increases because usually the first baroclinic mode and the barotropic mode well dominate the others (Hua and Haidvogel 1986). As a result, surface and interior modes should intersect at a horizontal wavenumber that is smaller for larger depths. Such a tendency is present in our results (see Fig. 5). This means that at intermediate depths, the surface mode dominates the intermediate scales and the interior mode dominates the smallest scales.

To further check the SQG diagnosis of the surface dynamics from the density, we have chosen to test the relation in physical space that links the relative vorticity to the density. Because the observed surface vorticity spectrum has a flat slope (deduced from the $k^{-2}$ velocity spectrum), this relation allows focus to be placed on the range from mesoscales to small scales. From the appendix, this relation in spectral space is

$$
\hat{\zeta}(m, n)=\frac{g}{N_{0} \rho_{0}} k \hat{\rho}(m, n),
$$

where $m, n$ are the zonal and meridional wavenumbers, respectively. Here, $k$ is again defined as $k^{2}=m^{2}+n^{2}$. In physical space, (3) becomes

$$
\zeta(x, y)=\frac{g}{N_{0} \rho_{0}} \int_{m} \int_{n} k \hat{\rho}(m, n) e^{i(m x+n y)} d n d m,
$$

where $i^{2}=-1$. The relative vorticity predicted from the surface density using (4) compares relatively well with that directly calculated from $u$ and $v$ (cf. Figs. 7a,b). A scatterplot of the observed relative vorticity and its prediction by (4), using averaging over grid intervals on the abscissa (Fig. 7c), reveals a first-order statistical agreement between the two fields. This indicates that velocity and density not only have identical spectral amplitudes (see Fig. 5a) but also identical spectral phases. As expected from the relatively large Rossby number, the flow in the surface layers is not entirely explained in terms of SQG balance. Indeed, the correlation between the two relative vorticity fields (deduced from $u$ and $v$ and reconstructed from the surface density) is only 0.8 [instead of 0.98 in the low-resolution results of Lapeyre and Klein (2006)] and the scatterplot (Fig. 7c) displays a nonnegligible dispersion from (4) for all vorticity values. Not surprisingly, this dispersion is much larger in the region where $\zeta$ amplitudes are close or larger than $f$. In regions where the flow exhibits a strong cyclonic asymmetry, the prediction of the vorticity from density is clearly underestimated. A more detailed examination of the differences between Figs. 7a,b shows that both some vorticity filaments and some large-scale vorticity structures are underestimated in some regions, whereas they are overestimated in others. This may indicate an effect of the small-scale ageostrophic divergent motions onto the largest scales. Despite these discrepancies, these results confirm, at least on a first order, that the SQG relation (3) is valid.

Thus, the velocity in the surface layers can be remarkably, on first order, diagnosed from only the surface density using SQG relations. This would suggest that the decomposition invoked in Lapeyre and Klein (2006) may still apply for ocean dynamics with a Rossby number on the order of one within the range between mesoscales and small scales. The discrepancies noted in Fig. 7c and the surface vortical asymmetries (not allowed by SQG) indicate, however, that in some aspects, the flow-time evolution is not driven by the SQG dynamics as it was in Lapeyre and Klein (2006). Furthermore, the slope of the surface velocity spectrum is steeper than $k^{-2}$ for wavenumbers larger than 100 . We have checked that this steeper slope is quite robust when the coefficient for horizontal mixing is changed. Such a steep slope is reminiscent of the differences between SQG and SG induced by frontal dynamics, which could affect the nonlinear interactions (as noted by Andrews and Hoskins 1978; Blumen 1978). All of these features indicate some departures of the surface dynamics from SQG dynamics, which need to be addressed.

\section{Departure of surface dynamics from SQG}

\section{a. Restratification}

From Hakim et al. (2002), one prominent departure from SQG of flows with a large Rossby number is the restratification in the upper layers. Such characteristics conspicuously emerge in our simulation, as shown by Fig. 1a. The comparison of the Brunt-Väisälä frequency profile at equilibrium with that at initial time, displayed by this figure, reveals a strong restratification in the first $300 \mathrm{~m}$, which is further compensated for by a weaker destratification in the ocean interior. This restratification corresponds to a surface temperature increase of almost $1^{\circ} \mathrm{C}$. To explain the mechanisms that trigger such departure from SQG, theoretical arguments of Lapeyre et al. (2006), based on Ertel PV conservation for flows with large a Rossby number, can be invoked. These authors indicate that it is the surface frontogenesis mechanisms, and mainly their associated divergent motions, that drive a restratification in the 
(a)

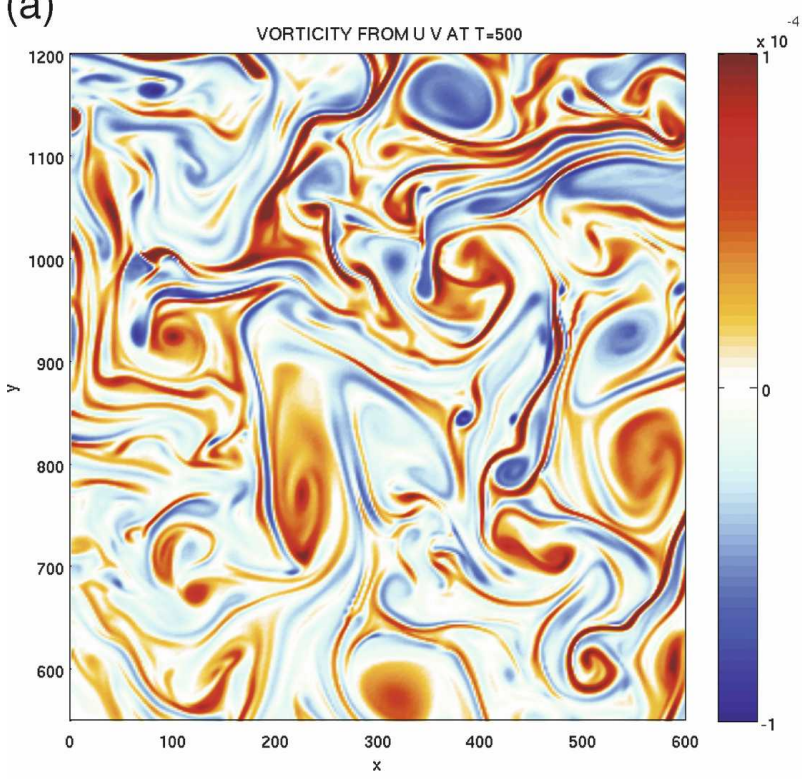

(b)

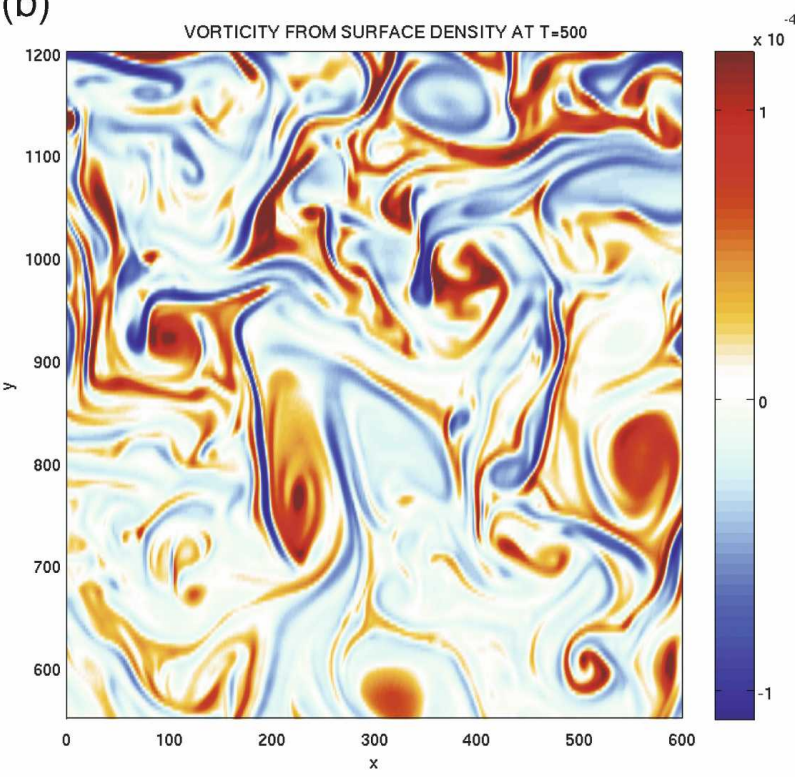

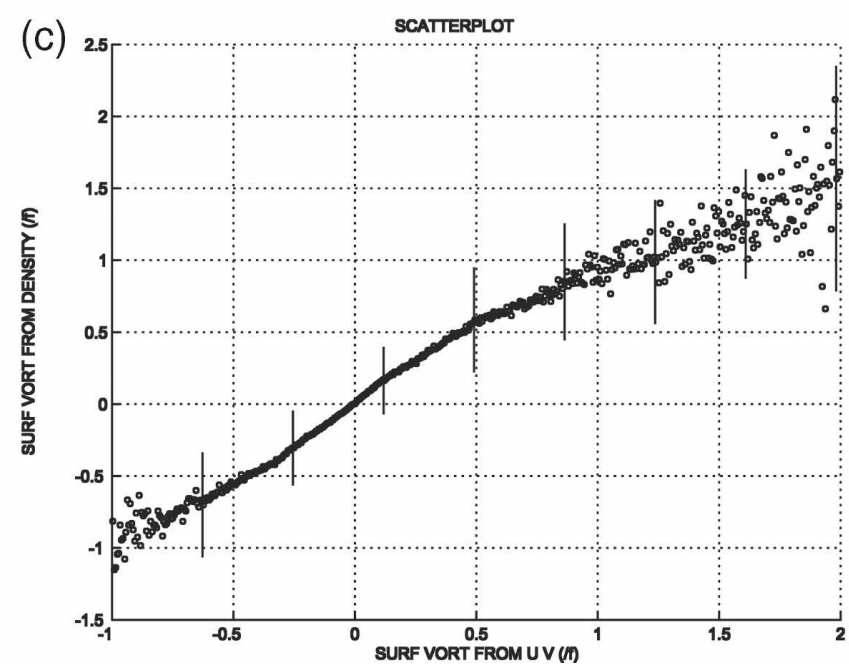

FIG. 7. Zoom of the surface relative vorticity field (a) deduced from $u$ and $v$ and (b) reconstructed from the surface density using (4); units in the $x$ and $y$ directions in are $\mathrm{km}$ and vorticity units are $\mathrm{s}^{-1}$. (c) Scatterplot between the original surface vorticity and its reconstruction from the surface density over the whole domain. In (c), each point represents the average over each grid interval on the abscissa (that has a total of 2000 grid intervals), and thin vertical lines show std dev around the averages.

surface layers. They show that this surface restratification competes with and actually inhibits the restratification in the interior through the vertical velocity.

Such strongly intensified surface frontogenesis mechanisms are present in our simulation. This is pointed out by the density spectra (Figs. 5a,d), which display larger amplitudes and shallower slopes at the surface than at depth. Indeed, the resulting density gradient spectrum (not shown) at the surface has a flat $\left(\approx k^{0}\right)$ slope with large amplitudes (as deduced from Fig. 5a), indicating strong density gradients at small scales. On the other hand, the steep $\left(\approx k^{-2.5}\right)$ slope of the density gradient spectrum at depth with smaller amplitudes (as deduced from Fig. 5d) indicates that density gradients there have larger scales and smaller amplitudes than at the surface. The large RMS value of the divergence of the horizontal motions (usually related to the thermal wind balance associated with the density gradients), reaching $0.025 f_{0}$ near the surface [and only $0.003 f_{0}\left(0.0012 f_{0}\right)$ at $\left.300 \mathrm{~m}(800 \mathrm{~m})\right]$, confirms the much stronger impact of the surface frontogenesis. Figure 8 furthermore emphasizes not only the larger amplitude of $w_{z}$ near the surface but also that $w_{z}$ is mostly captured by the small scales. 


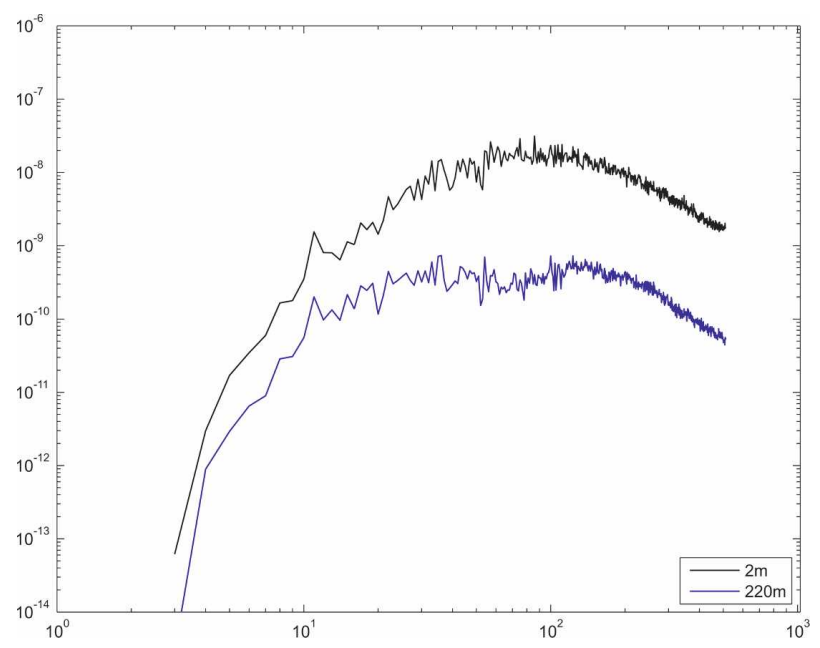

FIG. 8. Spectrum of the divergence $\left(w_{z}\right)$ near the surface (black curve) and at $220 \mathrm{~m}$ (blue curve). The horizontal axis displays the nondimensional wavenumber $k$. The value $k=10$ corresponds to a wavelength of $300 \mathrm{~km}$. Units on the vertical axis are $\mathrm{s}^{-2}$.

Because the restratification in the surface layers is compensated for by a destratification in the interior, the ageostrophic mechanisms near the surface implicitly affect the interior dynamics. Lapeyre et al. (2006) explain this interior destratification as a consequence of the effect of the mass conservation. The profile of the vertical velocity variance in the present simulation (Fig. $3 c$ ) displays a significant maximum at $200 \mathrm{~m}$ that corresponds to an RMS value of $10 \mathrm{~m}$. At $153 \mathrm{~m}$, the vertical velocity field (Fig. 9a) is characterized by smallscale patchy structures in the surface layers mostly located around eddies and within small-scale filaments. Its amplitude at this depth reaches quite large values: as large as $150 \mathrm{~m}$ per day. On the other hand, vertical sections (such as that on Fig. 9b) reveal that the vertical velocity forced near the surface by the frontogenesis extends down to depths as large as $900 \mathrm{~m}$ with larger horizontal scales. These noticeable features indicate a connection between the vertical velocity associated with the surface small-scale frontogenesis and the vertical velocity at depth related to mesoscale eddies. The large values of the divergent motions near the surface and the large depth extension of the vertical velocity field from the surface therefore suggest that this divergent field is a major dynamical component for the connection, at small scale, between the near-surface and interior dynamics.

The disappearance of the surface restratification and interior destratification when the resolution is $6 \mathrm{~km}$ confirms the small-scale character of the efficiency of these ageostrophic mechanisms. Furthermore, with the $6-\mathrm{km}$ resolution, the cyclonic-anticyclonic bias is no more present and the enstrophy (square of the vorticity) and vertical kinetic energy are decreased by a factor of 10 , whereas the horizontal kinetic energy in the upper layers decreases only by a factor of 2 .

\section{b. Surface energy and density fluxes}

One further departure from SQG, noted in section 3, was the steeper slope of the surface velocity spectrum observed in the small-scale range-reminiscent of the results of Andrews and Hoskins (1978) and Blumen (1978). Such characteristics may be a signature of the divergent motion effects on the nonlinear interactions. To check this, we have analyzed the impact of these motions on the nonlinear interactions in our simulation. For that purpose, the budget terms of the density variance and KE (with a focus on the latter) are estimated in spectral space.

The surface local budgets are

$$
\begin{aligned}
& \underbrace{\frac{1}{2} \frac{\partial|\hat{\rho}|^{2}}{\partial t}}_{d / d t}=\underbrace{-\operatorname{Re}\left[\hat{\rho}^{*}\left(\widehat{\left.\mathbf{u}_{h} \cdot \nabla_{H} \rho\right)}\right]\right.}_{\text {advection }}+\underbrace{\mathrm{MT}}_{\text {mixing }}, \\
& \underbrace{\frac{1}{2} \frac{\partial\left|\widehat{\mathbf{u}_{h}}\right|^{2}}{\partial t}}_{d / d t}=\underbrace{-\operatorname{Re}\left[\widehat{\mathbf{u}_{h}} * \cdot \widehat{\left(\mathbf{u}_{h} \cdot \nabla_{H} \mathbf{u}_{h}\right)}\right]}_{\text {advection }} \\
& \underbrace{-\frac{1}{\rho_{0}} \operatorname{Re}\left(\widehat{\mathbf{u}_{h}} * \cdot \widehat{\nabla_{H} p}\right)}_{\text {pressure }}+\underbrace{M T}_{\text {mixing }},
\end{aligned}
$$

where, again, $\mathbf{u}_{h}$ is the horizontal velocity field, $\nabla_{H}$ is the horizontal gradient operator, and $p$ is the pressure. Here, ()* stands for the complex conjugate and $\operatorname{Re}()$ is the real part. Advection terms involve the nonlinear terms of the momentum and density equations. Mixing terms include both vertical and horizontal mixing. All terms entering the momentum and density budget are stored online over a period of 30 days. We have, however, utilized the same method as in Capet et al. (2008c) to estimate the advection and horizontal mixing terms. Indeed, the upstream biased advection scheme used in ROMS includes an implicit mixing, which needs to be assessed. Thus, advection terms have been estimated using conservative centered differences. Then the implicit mixing is retrieved as the difference between the advection terms coming directly from ROMS and those estimated using conservative centered differences.

In the KE budget, the pressure gradient only involves the divergent part of the horizontal motions because the product of the rotational (or nondivergent) part by the pressure gradient is identically zero. It can be written as

$$
\widehat{\mathbf{u}_{h}} * \widehat{\nabla_{H}} p=\widehat{w_{z}} * \hat{p} .
$$


(a)

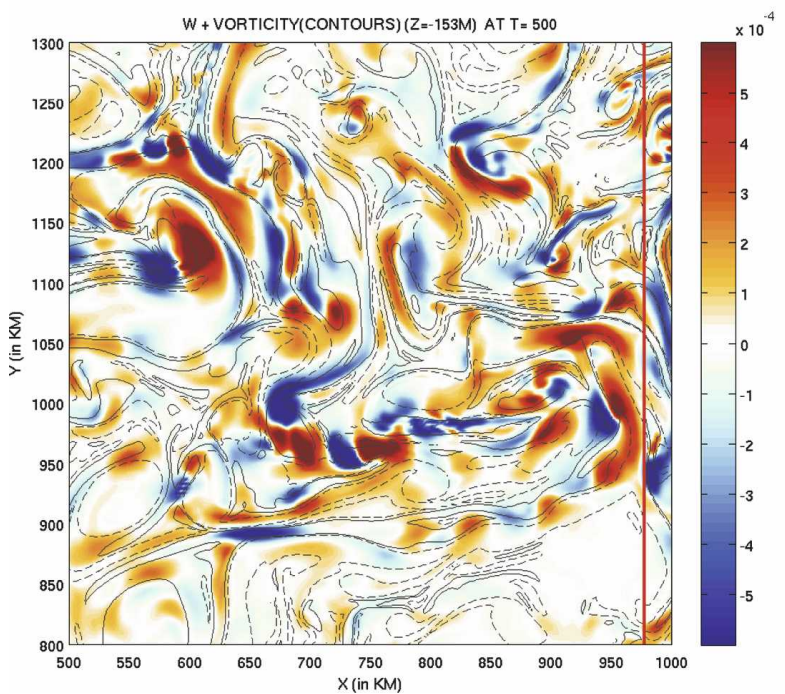

(b)

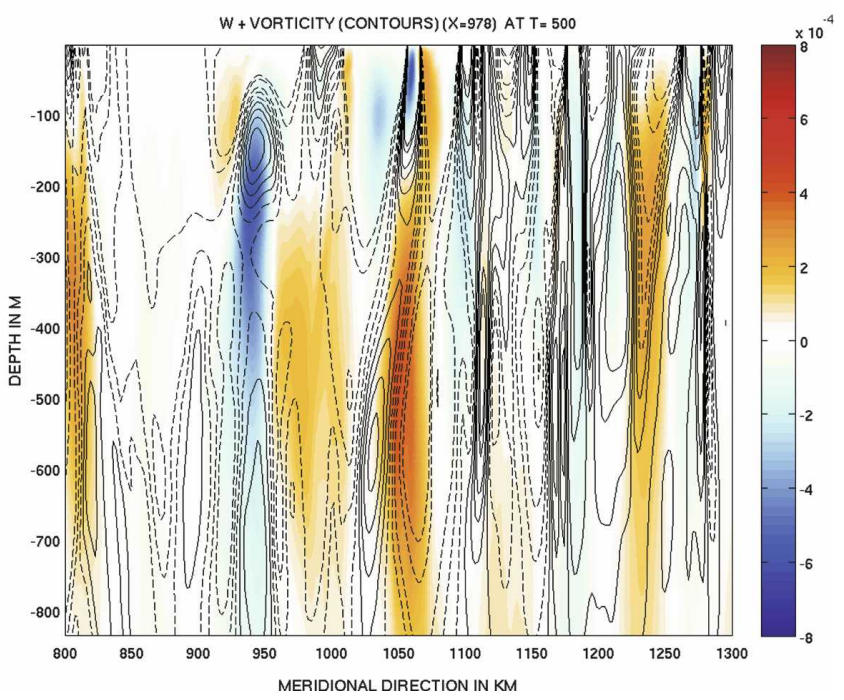

FIG. 9. (a) Vertical velocity field, $W$ (color), and relative vorticity (contours) at $153 \mathrm{~m}$; (b) vertical section of $W$ (color) and relative vorticity (contours). Location of the vertical section (b) in the horizontal plane is indicated by the red line in (a). Units for $W$ and the vorticity are $\mathrm{m} \mathrm{s}^{-1}$ and $\mathrm{s}^{-1}$, respectively.

The KE advection term

$$
\mathrm{KE}_{\mathrm{adv}}=-\operatorname{Re}\left[\widehat{\mathbf{u}_{h}} * \cdot\left(\widehat{\mathbf{u}_{h} \cdot \nabla_{H} \mathbf{u}_{h}}\right)\right.
$$

includes both a rotational part and a divergent part. If the velocity is decomposed as $\mathbf{u}_{h}=\mathbf{u}_{h_{r}}+\mathbf{u}_{h_{d}}$, with $\mathbf{u}_{h_{r}}$ being the rotational part and $\mathbf{u}_{h_{d}}$ the divergent part, then the rotational part of this term is

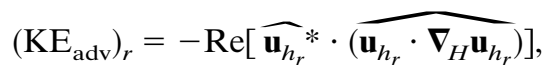

and the divergent part is

$$
\left(\mathrm{KE}_{\mathrm{adv}}\right)_{d}=\mathrm{KE}_{\mathrm{adv}}-\left(\mathrm{KE}_{\mathrm{adv}}\right)_{r} \text {. }
$$

This divergent part is absent in SQG because of the assumptions considered. Using the integrals in physical space,

$$
\begin{aligned}
& \int_{y} \int_{x} \mathbf{u}_{h_{r}} \cdot\left(\mathbf{u}_{h_{r}} \cdot \nabla_{H} \mathbf{u}_{h_{r}}\right) d x d y=0, \\
& \int_{y} \int_{x} \mathbf{u}_{h} \cdot\left(\mathbf{u}_{h} \cdot \nabla_{H} \mathbf{u}_{h}\right) d x d y=\int_{y} \int_{x}\left|\mathbf{u}_{h}\right|^{2} w_{z} d x d y,
\end{aligned}
$$

as well as the identity of the integrals in spectral and physical spaces, we get

$$
\begin{aligned}
\int_{0}^{\infty}\left(\mathrm{KE}_{\mathrm{adv}}\right)_{r}(k) d k & =0 \\
\int_{0}^{\infty} \mathrm{KE}_{\mathrm{adv}}(k) d k & =\int_{0}^{\infty}\left(\mathrm{KE}_{\mathrm{adv}}\right)_{d}(k) d k \\
& =\int_{0}^{\infty}\left(\widehat{\left|\mathbf{u}_{h}\right|^{2} w_{z}}\right)(k) d k .
\end{aligned}
$$

Relation (11) indicates that if the flow departs from SQG, the integral of the KE advection term may be nonzero because of the correlation between the ageostrophic divergence and the kinetic energy. The same reasoning can be applied to the density variance as noted by Blumen (1978).

Hereafter, we examine the integral budget for the kinetic energy and density variance. Their terms are just the integral of the local budget terms over the spectral wavenumber range $[k, \infty]$, that is,

$$
\Pi_{A}(k)=\int_{k}^{\infty} A(k) d k
$$

where $A$ is one of the terms in (5) and (6). The integral budget terms averaged over 30 days are shown on Figs. 10,11 . Those for the density variance have been normalized by the factor $g^{2} /\left(N_{0} \rho_{0}\right)^{2}$ used in section 3 for a better comparison with the KE budget terms. We mostly focus on the wavenumber range $(k>10)$, where velocity and density spectra are comparable. Foremost, the tendency terms of the two quantities (Fig. 10) are remarkably close, further supporting the similitude of surface density and velocity spectra. They are slightly positive, which is consistent with the tendency observed in Fig. $1 \mathrm{~b}$ between 495 and 525 days. We now examine in more detail the respective characteristics of these budgets with a stronger emphasis on the KE budget.

The integral surface density variance budget (Fig. 11a) reveals that the advection term contribution is positive, implying a forward nonlinear transfer (or cascade) of density variance for the range of wavenumbers 


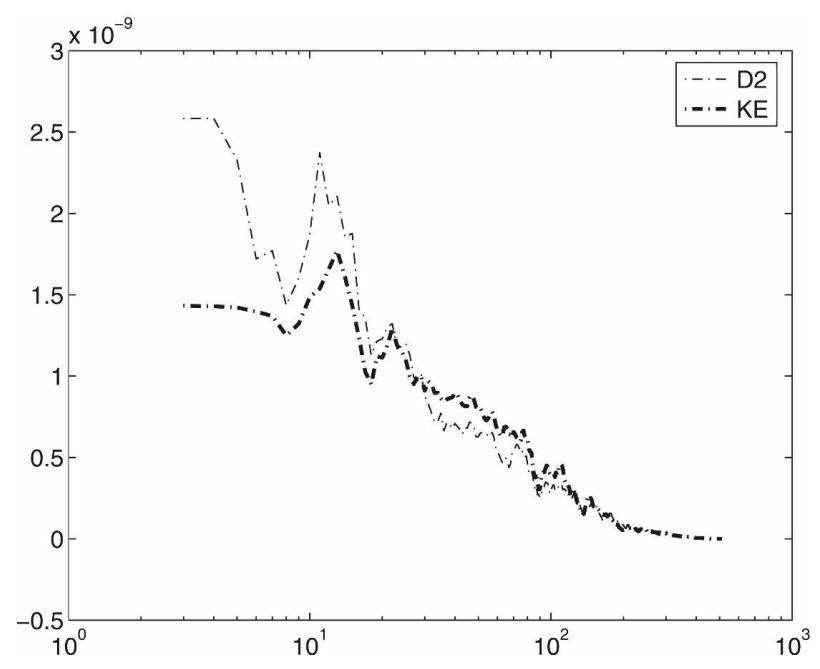

FIG. 10. Time rate of change for the surface KE and density variance (D2). The horizontal axis displays the nondimensional wavenumber $k$. The value $k=10$ corresponds to a wavelength of $300 \mathrm{~km}$. Units on the vertical axis are $\mathrm{m}^{2} \mathrm{~s}^{-3}$.

of interest $(k>10)$. This could be expected because the action of eddies is to stir the density field to small scales (at least to the deformation radius). The mixing term only partly compensates for the advection term. Mixing is principally dominated by horizontal mixing (vertical mixing has been found to be negligible).

The integral KE budget (Fig. 11b) is quite different from the density variance budget. The foremost difference is due to the advection term, which is mostly negative over the considered spectral range $(k>10)$. This indicates a dominant inverse nonlinear transfer (or cascade) of KE within the range between 300 and $30 \mathrm{~km}$. A noticeable, although small, direct KE cascade is present in the very small-scale range (scales smaller than $30 \mathrm{~km}$ ). The opposite advective cascades of density variance and $\mathrm{KE}$, within the mesoscale-small-scale ranges, may seem puzzling because of the local relationship between density variance and kinetic energy. This apparent paradox can be resolved if the other terms are examined. The pressure term (dashed black curve in Fig. 11b) is dominant in the KE budget and is positive over the whole spectral range. It partly compensates for the $\mathrm{KE}$ advective term and its maximum amplitude is twice as large as the latter. Because it is linked to the horizontal velocity divergence, its contribution as a source (especially within the small-scale range) indicates that it is mostly driven by small-scale frontogenesis. Next, the contribution of the mixing term to the integral KE budget is quite significant. Its amplitude at $k=20$ is as large as that of the KE advective flux. Furthermore, the vertical mixing contribution is now no longer negligible because it explains nearly half of the total KE mixing. At last, the tendency term is almost 10 times smaller than the dominant terms.

Thus, although density and velocity spectra are almost identical over a large spectral range (as confirmed by their tendency terms), their dynamics, as revealed by their budgets, are quite different. Such differences are also present in SQG [see Capet et al. (2008a, hereafter CAP] and can be clarified on a first order in terms of (a)

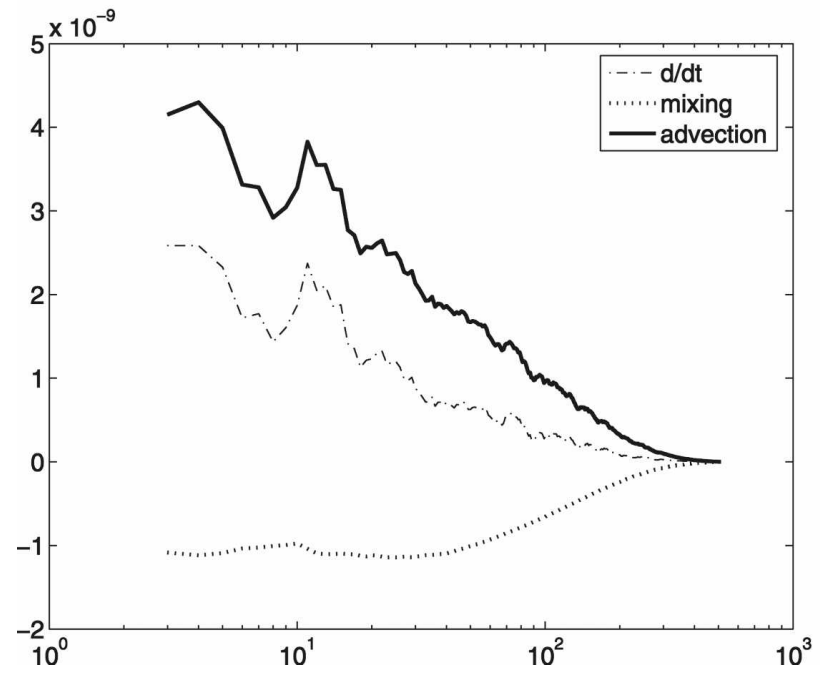

(b)

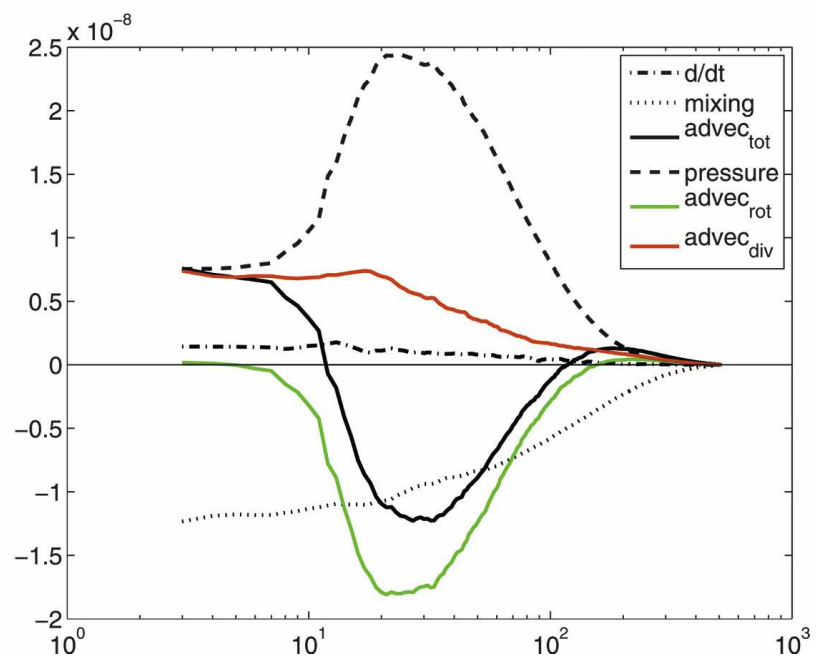

FIG. 11. Integral budget for (a) the surface density variance [normalized by $\left(g^{2} / N^{2} \rho_{0}^{2}\right)$ ] and (b) surface KE. The horizontal axis displays the nondimensional wavenumber $k$. The value $k=10$ corresponds to a wavelength of $300 \mathrm{~km}$. Units on the vertical axis are $\mathrm{m}^{2} \mathrm{~s}^{-3}$. 
QG frontogenesis (CAP). On the one hand, the density variance experiences a dominant forward cascade, and therefore small-scale surface density gradients (i.e., surface fronts) rapidly develop. On the other hand, to preserve the cross-front geostrophic balance, the alongfront surface velocity must accelerate. This acceleration, which represents a KE increase is produced by the ageostrophic (or divergent) part of the horizontal motions, which leads to a positive correlation between divergence and pressure (CAP). The contribution of the small scales to the positiveness of this pressure term is determinant. As a result, this term is compensated for by the KE advective term (solid black curve in Fig. $11 b$ ), which is principally negative.

The departure from SQG in our simulation is highlighted when the $\mathrm{KE}$ advection flux is split into rotational and divergent parts. The rotational part of the $\mathrm{KE}$ advective flux (displayed by the green curve in Fig. $11 b)$ is principally negative over the whole spectral range and, as expected from (10), returns to zero at the largest scales. It displays a direct cascade in the smallscale range, but the cascade is much weaker than that displayed by the total $\mathrm{KE}$ advective flux. These significant differences with the total $\mathrm{KE}$ advective flux are explained by the divergent part (which would be zero in SQG) represented by the red curve in Fig. $11 \mathrm{~b}$ and whose magnitude quantifies the departure of the flow dynamics from SQG. This part is positive over the whole spectral range. It is characterized by a positive increase within the small-scale range and a plateau within the large-scale range, with a magnitude almost half the maximum amplitude of the rotational part. Such behavior indicates that the correlation between the divergence and the kinetic energy at small scale significantly affects the total KE advective flux over the whole spectral range. This highlights the strong impact of the small-scale frontal structures (with large Rossby numbers) on the whole spectral range through the nonlinear interactions. One noticeable feature is that the magnitude of the pressure term at the largest scales is similar to that of the divergent part of the advection flux (Fig. 11b). So far we have no explanation for this feature. The departure from SQG of the pressure term has not been quantified. However, Capet et al. (2008c) clearly shows that within the small-scale range, the magnitude of this term increases as the resolution increases. This indicates that the divergent motions associated with small-scale fronts should also affect the pressure term when the Rossby number is large.

These frontogenesis mechanisms also explain the different mixing contributions. Horizontal mixing acts to halt the horizontal stirring of density at very small scale. On the other hand, the additional contribution of ver- tical mixing to the KE budget acts to halt the growth of the vertical shear of horizontal motions in frontal structures. Thus, although mixing coefficients (section 2a) have been chosen as small as possible, the mixing contribution is nonnegligible and actually helps prevent both the thermal wind imbalance allowed in primitive equations and the development of singularities near the surface (see also Boyd 1992).

All of these results are consistent with and in some ways extend those of CAP, Molemaker et al. (2008, manuscript submitted to J. Fluid Mech.), and Capet et al. (2008c). They indicate that divergent motions play an important role in the nonlinear transfers between scales for kinetic energy on a large range of scales. The corresponding energy and density budgets for deeper levels will be reported in another forthcoming work.

\section{Conclusions}

Our high-resolution simulation highlights that submesoscale structures, intensified in the upper oceanic layers, are the major component of a specific surface dynamical mode that competes with the interior mode. Ageostrophic (or finite time) surface frontogenesis underlies the physics that drive the submesoscale structures. As a result, dynamics in the surface layers involve large Rossby numbers, a significant cyclone-anticyclone asymmetry, and a strong surface restratification. This restratification is compensated for by a destratification in the ocean interior, which is interpreted in terms of an interaction between the surface and interior modes driven by the small-scale divergent motions. A surface KE budget analysis reveals that the ageostrophic divergent motions associated with smallscale surface frontogenesis significantly alter the nonlinear advective transfers over the whole spectral range. This means that advection of momentum quantities, and therefore their time evolution, is driven not only by the geostrophic velocities but also by the ageostrophic divergent velocities. This is this ageostrophic advection that produces the significant vortical asymmetries and the surface restratification not allowed by the SQG theory.

But, despite this strong ageostrophic character, some of the main surface properties are surprisingly still close to the SQG equilibrium. They include a noticeable shallow $\left(\approx k^{-2}\right.$ ) velocity spectrum as well as a conspicuous local spectral relationship between surface kinetic energy, $\mathrm{SSH}$, and density variance over a large range of scales (from 400 to $4 \mathrm{~km}$ ). As a consequence, surface velocities can be remarkably diagnosed from only the surface density using SQG relations. This suggests that the validity of these specific SQG properties extends to 
dynamical regimes involving larger Rossby numbers. In some ways, the picture that emerges from these results resembles that involved in semigeostrophic theory, for example, which allows large Rossby numbers: the field is approximately in geostrophic equilibrium, but its time evolution is driven by both the geostrophic and ageostrophic velocities (this last characteristic explains the departure of SG theory from QG theory).

As such, these findings reconcile and extend those of Lapeyre and Klein (2006) - related to the decomposition of the flow into SQG and QG modes-and those of Lapeyre et al. (2006) - about the role of vertical velocity in the restratification-destratification process. The surface-interior interaction appears to be a two-way interaction. On one hand, the interior dynamics affect the large scales of the dynamics in the surface layers, a result already obtained within the SQG-QG framework (Lapeyre and Klein 2006). On the other hand, the present study confirms that near-surface dynamics affect the interior dynamics through the divergent motions associated with the small-scale surface frontogenesis mechanisms. Indeed, the quite deep extension of the vertical velocity triggered by surface frontogenesis and the role assigned to this velocity in the restratification/destratification process [as shown in Lapeyre et al. (2006)] indicate that vertical velocity is a major component for the communication between the surface and interior modes.

These results provide an answer to the questions mentioned in the introduction. Indeed, they strongly emphasize the necessity to explicitly take into account the small-scale frontogenesis mechanisms and the related divergent motions in the upper layers to accurately represent the nonlinear interactions and therefore the properties of an oceanic turbulent eddy field. A high spatial resolution, consistent in both the horizontal and vertical directions, is therefore required to represent the dynamics of these small-scale structures.

No atmospheric forcing has been considered here, essentially for a better understanding of the specific properties of the near-surface dynamics relative to the interior dynamics. However, the importance of the vertical velocity related to the surface frontogenesis revealed by these results suggests that in presence of atmospheric forcings, mechanisms such as those described by Thomas (2005), Boccaletti et al. (2007), and Capet et al. (2008b) may substantially affect the upperlayer dynamics. A more detailed study is needed to understand the specific impact of small-scale divergent motions at depth. This will be reported in a forthcoming work. A last comment concerns a notable consequence related to the statistical relationship between SSH and surface density. Such property could be exploited for the analysis and interpretation of altimeter data jointly with high-resolution SST data (see Isern-Fontanet et al. 2006).

Acknowledgments. This work is supported by IFREMER and CNRS (France). Numerical simulations reported here were done on the Earth Simulator (Yokohama, Japan), whose access has been possible through a M.O.U. signed between IFREMER, CNRS, and JAMSTEC. PK, BLH, and SLG also acknowledge the support from the French ANR (Agence Nationale pour la Recherche, Contract ANR-05-CIGC-010). We thank Guillaume Roullet for useful comments and both reviewers for their very constructive suggestions and questions.

\section{APPENDIX}

\section{SQG Diagnosis}

Within the QG framework, SQG theory in addition considers a nonzero surface density and assumes a uniform potential vorticity in the interior. Furthermore, the flow is supposed to decay from the surface (i.e., the streamfunction $\psi \rightarrow 0$ as $z \rightarrow-\infty)$. The resulting PV equation is

$$
\nabla_{H}^{2} \psi+\partial_{z} \frac{f_{0}^{2}}{N_{0}^{2}} \partial_{z} \psi=0
$$

with

$$
-\left.\partial_{z} \psi\right|_{z=0}=\frac{g}{f_{0} \rho_{0}} \rho_{s} .
$$

Here, $\rho_{s}$ is the surface density and $\nabla_{\mathrm{H}}$ is the horizontal gradient operator. Solving these two equations in the Fourier space, assuming $N_{0}$ is constant, leads to

$$
\hat{\psi}(m, n, z)=-\frac{g}{N_{0} \rho_{0}} \frac{\hat{\rho}_{s}(m, n)}{k} \exp \left(k \frac{N_{0}}{f_{0}} z\right),
$$

where $\left({ }^{\wedge}\right)$ is the horizontal spectral transform, $m, n$ are the zonal and meridional wavenumbers, and $k=\left(m^{2}+\right.$ $\left.n^{2}\right)^{1 / 2}$. Then, using the classical QG relations, the dynamics at any depth are entirely expressed in terms of the surface density, $\rho_{s}$ :

$$
\begin{aligned}
u & =-\partial_{y} \psi, \\
v & =\partial_{x} \psi, \\
\hat{\rho}(m, n, z) & =\hat{\rho}_{s}(m, n) \exp \left(\frac{N_{0} k z}{f_{0}}\right) .
\end{aligned}
$$

This property directly comes from the uniform potential vorticity assumption. The following spectral relation can be also deduced:

$$
\hat{\zeta}=\frac{g}{N_{0} \rho_{0}} k \hat{\rho} .
$$


Furthermore, from (A3), (A4), and (A5), the relation between the kinetic energy and the density variance at any depth is

$$
\mathrm{KE}_{h}(k, z) \approx \frac{g^{2}}{N_{0}^{2} \rho_{0}^{2}}|\hat{\rho}(k, z)|^{2} .
$$

\section{REFERENCES}

Abraham, E. R., C. S. Law, P. W. Boyd, S. J. Lavender, M. T. Maldonado, and A. R. Bowle, 2000: Importance of stirring in the development of an iron-fertilized phytoplankton bloom. Nature, 407, 727-730.

Andrews, D. G., and B. J. Hoskins, 1978: Energy spectra predicted by semi-geostrophic theories of frontogenesis. J. Atmos. Sci., 35, 509-512.

Blumen, W., 1978: Uniform potential vorticity flow. Part I: Theory of wave interactions and two-dimensional turbulence. J. Atmos. Sci., 35, 774-783.

Boccaletti, G., R. Ferrari, and B. Fox-Kemper, 2007: Mixed layer instabilities and restratification. J. Phys. Oceanogr., 37, 22282250.

Boyd, J. P., 1992: The energy spectrum of fronts: Time evolution of shocks in Burgers' equation. J. Atmos. Sci., 49, 128-139.

Capet, X., P. Klein, B. Hua, G. Lapeyre, and J. C. McWilliams, 2008a: Surface kinetic energy transfer in surface quasigeostrophic flows. J. Fluid Mech., 604, 165-174.

_ J. C. McWilliams, M. J. Molemaker, and A. F. Shchepetkin, 2008b: Mesoscale to submesoscale transition in the California current system. Part II: Frontal processes. J. Phys. Oceanogr., 38, 44-64.

,-- , and $-2008 \mathrm{c}$ : Mesoscale to submesoscale transition in the California current system. Part III: Energy balance and flux. J. Phys. Oceanogr., in press.

Charney, J., 1971: Geostrophic turbulence. J. Atmos. Sci., 28, 1087-1095.

Haine, T. W., and J. Marshall, 1998: Gravitational, symmetric, and baroclinic instability of the ocean mixed layer. J. Phys. Oceanogr., 28, 634-658.

Hakim, G. J., C. Snyder, and D. J. Muraki, 2002: A new surface model for cyclone-anticyclone asymmetry. J. Atmos. Sci., 59, 2405-2420.

Held, I. M., and V. D. Larichev, 1996: A scaling theory for horizontally homogeneous, baroclinically unstable flow on a beta plane. J. Atmos. Sci., 53, 946-952.

_ R. T. Pierrehumbert, S. T. Garner, and K. L. Swanson, 1995: Surface quasi-geostrophic dynamics. J. Fluid Mech., 282, $1-20$.

Hoskins, B. J., 1975: The geostrophic momentum approximation and the semigeostrophic equations. J. Atmos. Sci., 32, 233242.

Hua, B. L., 1994: Skewness of the generalized centrifugal force divergence for a joint normal distribution of strain and vorticity components. Phys. Fluids, A6, 3200-3202.

— , and D. B. Haidvogel, 1986: Numerical simulations of the vertical structure of quasigeostrophic turbulence. J. Atmos. Sci., 43, 2923-2936.

_ J. C. McWilliams, and P. Klein, 1998: Lagrangian accelerations in geostrophic turbulence. J. Fluid Mech., 366, 87-108.

Hurlburt, H. E., and P. J. Hogan, 2000: Impact of $1 / 8^{\circ}$ to $1 / 64^{\circ}$ resolution on Gulf Stream model-data comparisons in basin- scale subtropical Atlantic Ocean models. Dyn. Atmos. Oceans, 32, 283-329.

Isern-Fontanet, J., B. Chapron, G. Lapeyre, and P. Klein, 2006: Potential use of microwave sea surface temperatures for the estimation of ocean currents. Geophys. Res. Lett., 33, L24608, doi:10.1029/2006GL027801.

Juckes, M., 1994: Quasigeostrophic dynamics of the tropopause. $J$. Atmos. Sci., 51, 2756-2768.

Karsten, R., H. Jones, and J. Marshall, 2002: The role of eddy transfer in setting the stratification and transport of a circumpolar current. J. Phys. Oceanogr., 32, 39-54.

Klein, P., A. Tréguier, and B. L. Hua, 1998: Three-dimensional stirring of thermohaline fronts. J. Mar. Res., 56, 589-612.

Lapeyre, G., and P. Klein, 2006: Dynamics of the upper oceanic layers in terms of surface quasigeostrophy theory. J. Phys. Oceanogr., 36, 165-176.

,-- , and B. L. Hua, 2006: Oceanic restratification by surface frontogenesis. J. Phys. Oceanogr., 36, 1577-1590.

Large, W. G., S. C. Doney, and J. C. McWilliams, 1994: Oceanic vertical mixing: A review and a model with a nonlocal boundary layer parameterization. Rev. Geophys., 32, 363-403.

Larichev, V. D., and I. M. Held, 1995: Eddy amplitudes and fluxes in a homogeneous model of fully developed baroclinic instability. J. Phys. Oceanogr., 25, 2285-2297.

McWilliams, J. C., 1984: The emergence of isolated coherent vortices in turbulent flow. J. Fluid Mech., 146, 21-43.

, 1985: Submesoscale coherent vortices in the ocean. Rev. Geophys., 23, 165-182.

_ 1989: Statistical properties of decaying geostrophic turbulence. J. Fluid Mech., 198, 199-230.

Rhines, P. B., 1979: Geostrophic turbulence. Annu. Rev. Fluid Mech., 11, 404-441.

Rivière, P., A.-M. Tréguier, and P. Klein, 2004: Effects of bottom friction on nonlinear equilibration of an oceanic baroclinic jet. J. Phys. Oceanogr., 34, 416-432.

Salmon, R. S., 1980: Baroclinic instability and geostrophic turbulence. Geophys. Astrophys. Fluid Dyn., 15, 167-211.

Scott, R. K., 2006: Local and nonlocal advection of a passive scalar. Phys. Fluids, 56, 122-125.

Shchepetkin, A. F., 2008: Computational kernel algorithms for fine-scale, multi-process, long-time oceanic simulations. Handbook of Numerical Analysis: Special Volume: Computational Methods for the Atmosphere and the Oceans, R. Teman and J. Tribbia, Eds., in press.

- and J. C. McWilliams, 2005: The regional oceanic modeling system (ROMS): A split-explicit, free-surface, topographyfollowing-coordinate ocean model. Ocean Modell., 9, 347-404.

Siegel, A., J. B. Weiss, J. Toomre, J. C. McWilliams, P. S. Berloff, and I. Yavneh, 2001: Eddies and vortices in ocean basin dynamics. Geophys. Res. Lett., 28, 3183-3186.

Smith, K. S., and G. K. Vallis, 2001: The scales and equilibration of midocean eddies: Freely evolving flow. J. Phys. Oceanogr., 31, 554-571.

Snyder, C., W. C. Skamarock, and R. Rotunno, 1991: A comparison of primitive-equation and semigeostrophic simulations of baroclinic waves. J. Atmos. Sci., 48, 2179-2194.

Thomas, L. N., 2005: Destruction of potential vorticity by winds. $J$. Phys. Oceanogr., 35, 2457-2466.

Tulloch, R., and K. S. Smith, 2006: A theory for the atmospheric energy spectrum: Depth-limited temperature anomalies at the tropopause. Proc. Natl. Acad. Sci. USA, 103, 14 69014694. 